\title{
INTERCORRÊNCIA AUDIOLÓGICA EM MÚSICOS APÓS UM SHOW DE ROCK
}

\author{
Hearing incidents in musicians after a rock concert
}

\author{
Marcela Pfeiffer ${ }^{(1)}$, Rita Leniza Oliveira da Rocha $^{(2)}$, Fernanda Ribeiro de Oliveira $^{(3)}$, Silvana Frota $^{(4)}$
}

\begin{abstract}
RESUMO
Objetivo: verificar mudança temporária do limiar de audição de músicos, após exposição a níveis de pressão sonora elevados de um show de rock. Métodos: foi utilizada uma amostra com seis músicos componentes da banda. Foram feitos: anamnese ocupacional, determinação dos níveis mínimos de audição e reflexos acústicos, antes e após o show de rock. A mensuração do ruído da sala do exame e do palco foi realizada por meio de um dosímetro. Os resultados foram comparados e analisados estatisticamente, utilizando-se o teste $t$ Student, com critério para determinação de significância de $p$ maior do que 0,05 . Resultados: para a dosimetria, foi encontrada a exposição ao ruído do show com valor de LAVG igual a $98,5 \mathrm{~dB}$. Dentre os aspectos comportamentais relacionados ao ruído, o zumbido foi a queixa mais presente entre os integrantes. $\mathrm{Na}$ audiometria tonal, as maiores diferenças pré e pós-exposição, foram encontradas nas freqüências altas, sendo a orelha direita a que apresentou maiores mudanças temporárias de limiar. Os resultados foram significantes nas freqüências de $2000,3000,4000$ e $6000 \mathrm{~Hz}$, na orelha direita $(p=2,7 ; p=2,59$; $p=3,7$ e $p=2,86)$ e, na freqüência de $4000 \mathrm{~Hz}$, na orelha esquerda $(p=2,87)$. Na medida do reflexo acústico após o show, a orelha direita obteve o maior índice de ausência de reflexo, com o índice de $40 \%$; entretanto, foram encontradas diferenças estatisticamente significantes, na presença de reflexo acústico na comparação pré e pós-exposição, apenas na orelha esquerda $(p=3,64)$. Conclusão: músicos expostos a níveis de pressão sonora intensos apresentaram alteração temporária do limiar e alteração do reflexo acústico.
\end{abstract}

DESCRITORES: Audiologia; Ruído Ocupacional; Testes Auditivos; Perda Auditiva

\section{INTRODUÇÃO}

O ruído é um dos principais agentes físicos presentes nos ambientes de trabalho ${ }^{1}$, em diversos tipos de instalações ou atividades profissionais. Por sua enorme ocorrência e visto que os efeitos à saúde dos indivíduos expostos são consideráveis, torna-se um dos maiores focos de atenção por parte dos higi-

(1) Fonoaudióloga; Audiologista da Clínica Check-up; Especialização em Audiologia pelo CEFAC - Saúde e Educação.

(2) Fonoaudióloga do Hospital Central Aristarcho Pessoa; Especialização em Audiologia pelo CEFAC - Saúde e Educação.

(3) Fonoaudióloga; Audiologista do Grupo Mil; Especialização em Audiologia pelo CEFAC - Saúde e Educação.

(4) Fonoaudióloga; Professora da Universidade Federal do Rio de Janeiro; Doutora em Distúrbios da Comunicação Humana pela Universidade Federal de São Paulo. enistas e profissionais da segurança e saúde do trabalhador.

Todos os sons têm potencial de serem descritos como ruído. A classificação do ruído é subjetiva e sua distinção se refere ao fato de ser ou não desejável. Todavia, a denominação ruído é utilizada para descrever um sinal acústico aperiódico, originado da superposição de vários movimentos de vibração, com diferentes freqüências, as quais não apresentam relação entre $\mathrm{si}^{2}$.

Vivendo nas grandes cidades, torna-se muito difícil não se expor a situações de ruído intenso e, certamente, em algum momento da vida, os seus efeitos aparecerão. Seja nas ruas, em festas, nas fábricas, em ambientes barulhentos, em shows de música, além da exposição às drogas ototóxicas, aumentando ou facilitando a perda auditiva induzida por ruído ${ }^{3}$.

A música é considerada como um som agradável e pode estar associada a fatos importantes ocorridos 
em nossas vidas; só que, quando usada de forma intensa, pode transformar-se em um transtorno na saúde das pessoas ${ }^{4}$. Sons desejados (prazerosos), como a música, são menos perigosos do que os sons não desejados (como o ruído industrial) ${ }^{5}$, mas nem por isso deixam de ser um fator de risco para perdas auditivas.

O ouvido humano é capaz de suportar sons dentro de uma faixa de 0 a $90 \mathrm{~dB}$ NPS. Acima disto, os sons se tornam desconfortáveis e dolorosos, podendo ser lesivos e destrutivos ao se aproximarem de 130 dB NPS ${ }^{6}$. Estudos mostram que os grandes grupos de rock utilizam equipamentos de amplificação, com potências de milhares de watts, ultrapassando os 120 dB NPS junto às caixas acústicas ${ }^{7}$ e, por esta razão, é possível classificar os músicos de uma banda de rock como sendo profissionais com risco de perda auditiva, assim como os ouvintes dos shows ${ }^{8}$.

Apesar de apresentarem, na maioria das vezes, resposta normal nas audiometrias, não pode ser afastada a possibilidade de uma futura perda auditiva, pois, antecedendo a ocorrência da mudança permanente de limiar, tem-se observado sempre uma mudança temporária, atingindo o seu máximo, logo após o término da exposição ${ }^{2}$. Esta mudança temporária também dominada de Temporary Threshold Shift (TTS), se manifesta devido a discretas alterações intracelulares, edema das terminações nervosas junto às células ciliadas, alterações vasculares, químicas e exaustão metabólica, além da diminuição da rigidez dos estereocílios, que ocasionam uma redução na capacidade das células em perceberem a energia sonora que as atingem ${ }^{9}$.

Essas alterações podem ser reversíveis, mas dependendo da intensidade do ruído (música) e duração da exposição do indivíduo a ela, podem desencadear as Perdas Auditivas Induzidas por Ruído (PAIR) ${ }^{10}$, classificadas como qualquer alteração dos limiares auditivos, do tipo neurossensorial, decorrente de exposição sistemática a níveis de pressão sonora elevados. É uma perda auditiva irreversível, progredindo com o tempo de exposição ao ruído, tendo como característica básica uma alteração primária nos limiares entre 3000 e $6000 \mathrm{~Hz}^{11}$.

A Perda Auditiva Induzida por Ruído, primeiramente, é conseqüência de uma lesão em nível de receptores sensoriais da cóclea, isto é, nas células ciliadas externas do órgão de Corti. Em alguns casos, as células de sustentação também podem ser afetadas. Dependendo do estímulo (intensidade, duração, freqüência, horário), o ruído pode causar dano às células ciliadas que podem ser, de forma mais leve, lesões em algumas de suas estruturas e, de forma mais severa, sua destruição total ${ }^{12}$.
Além disso, a exposição contínua ao ruído pode causar alterações cardiovasculares, neuroendócrinas, imunológicas, gastrointestinais, como também, afetar o sono ${ }^{13}$, além do aparecimento do zumbido e dificuldade de discriminar os sons da fala.

O teste do reflexo acústico é uma técnica de extrema importância para a audiologia clínica. É um exame objetivo e rápido, que registra a contração dos músculos da orelha média, na presença de um som de forte intensidade. O limiar do reflexo acústico corresponde à menor intensidade de um som capaz de desencadear o mecanismo de proteção da orelha média frente a sons intensos. Em 1954, este reflexo foi considerado um mecanismo de proteção para a orelha interna, de danos que poderiam ser causados por sons intensos ${ }^{14}$.

A relação entre as propriedades do reflexo acústico e a mudança temporária de limiar, não tem sido muito estudada. Em 1983, foi realizado um estudo o qual mostrou a mudança do reflexo acústico após a exposição ao ruído, aumentando o índice de ausência do reflexo após a exposição ${ }^{15}$.

Ė possível afirmar que a audiologia está voltando os seus esforços para a proteção da audição dos funcionários industriais, deixando de lado a importância do trabalho preventivo com músicos, pois não pode esquecer que a perda auditiva ocupacional é irreversível, mas possível de ser evitada ${ }^{16}$.

Este trabalho tem como objetivo verificar a mudança temporária do limiar de audição em músicos expostos a níveis de pressão sonora elevados em um show de rock.

\section{MÉTODOS}

A pesquisa foi realizada em uma casa de shows, localizada na cidade do Rio de Janeiro, em uma sala com ruído constante.

Foram avaliados seis músicos, componentes de uma banda de "rock", do sexo masculino, com idades variando entre 20 e 30 anos.

Foram realizadas duas medidas de dosimetria, com o posicionamento do dosímetro, no meio do palco, no qual os músicos se apresentaram. A primeira, antes da pesquisa dos limiares auditivos, com o objetivo de avaliar a intensidade de ruído na sala dos exames. Os valores encontrados foram de $72,5 \mathrm{~dB}$, na sala de exames antes do show e 73,8 $\mathrm{dB}$ depois do show, comprovando, assim, que o ruído ambiental não influenciou a mudança dos limiares auditivos dos músicos.

Também, foi realizada a dosimetria para verificar os níveis de pressão sonora do show de rock, tendo sido encontrada uma intensidade de $98,5 \mathrm{~dB}$.

Os músicos responderam a um questionário com perguntas referentes à: idade, passado otológico e 
zumbido, a fim de caracterizar a amostra e excluir indivíduos com problemas otológicos anteriores.

Foi realizada a meatoscopia, antes da avaliação audiométrica, a fim de descartar alterações da membrana timpânica, presença de corpos estranhos ou acúmulo de cerume no meato acústico externo.

A pesquisa constou de determinação dos níveis mínimos de audição e medidas da imitância acústica, realizadas antes e após a exposição ao ruído do show, sendo que a avaliação após o show foi realizada, no máximo, 30 minutos após o término da exposição.

A determinação dos níveis mínimos de audição, pré e pós-exposição, foi realizada por via aérea, nas freqüências de $500 \mathrm{~Hz}$ a $8000 \mathrm{~Hz}$, utilizando audiômetro da marca Danplex DA-74, com o uso dos fones de atenuação sonora da marca Amplaid, sem uso de cabina acústica. O audiômetro foi calibrado de acordo com a norma ISO 8253-1, 1989.

$\mathrm{Na}$ imitanciometria, foi realizada a pesquisa da timpanometria, apenas antes do show, com o objetivo de excluir da pesquisa os portadores de curvas dos tipos B (curva característica de indivíduos com secreção na orelha média) e tipo $C$ (curva característica de indivíduos com disfunção tubária) ${ }^{17}$.

A pesquisa do reflexo acústico, pré e pós-exposição, realizou-se nas freqüências de $500 \mathrm{a} 4000 \mathrm{~Hz}$, no modo ipsilateral, utilizando o imitanciômetro Handtymp.

A presente pesquisa foi avaliada pelo Comitê de Ética em Pesquisa do CEFAC - Saúde e Educação, sob n $128 / 04$, tendo sido considerada como sem risco e com necessidade do consentimento livre e esclarecido.
Após a realização de todos os testes, os resultados da determinação dos níveis mínimos de audição e do reflexo acústico, pré e pós-exposição ao show, foram comparados, e analisados estatisticamente, utilizando o teste $t$ Student. O critério para determinação de significância foi $5 \%$. Foram assinalados com asterisco os valores considerados significantes.

\section{RESULTADOS}

Durante a anamnese, foi averiguada a presença do zumbido em quatro músicos, ou seja, em $67 \%$ deles (Figura 1). Além disso, as queixas do aparecimento do zumbido foram mais freqüentes, após a exposição ao ruído do show de rock (Figura 2 ).

Na Figura 3, encontram-se os níveis mínimos de audição em dB por freqüência, antes e depois da exposição ao ruído, sendo possível observar a média dos limiares de audibilidade, antes e após exposição, respectivamente, para as orelhas: direita e esquerda.

Por meio desta análise, foi possível observar diferenças significantes na orelha direita para as freqüências de 500, 2000, 3000, 4000 e 6000 Hz, e para a freqüência de $4000 \mathrm{~Hz}$, na orelha esquerda.

Na Figura, 4 é possível verificar a presença e a ausência do reflexo acústico, antes e após a exposição. Pode-se observar um aumento de ausência dos reflexos acústicos, após o término do show, sendo esta diferença estatisticamente significante na orelha esquerda.

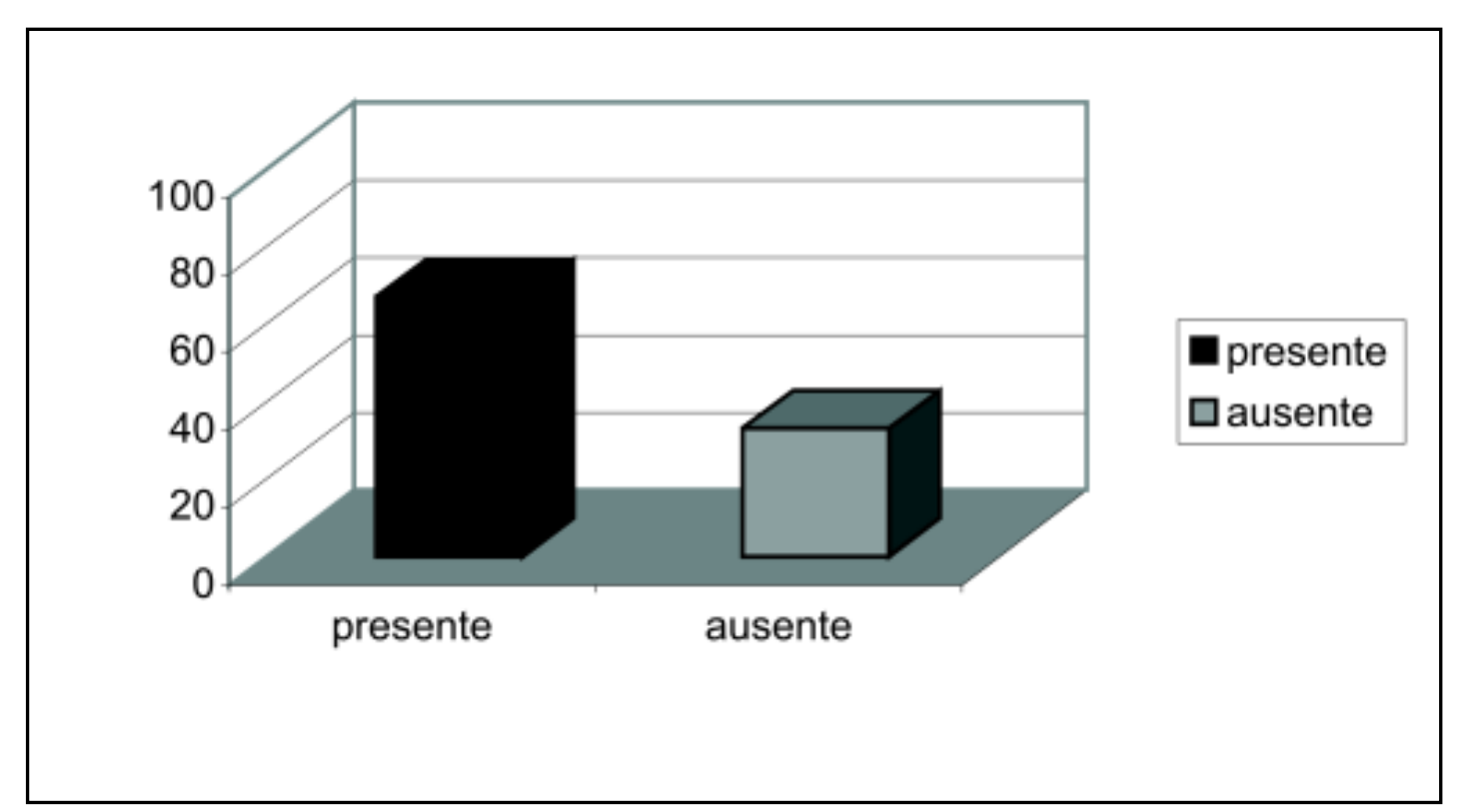

Figura 1 - Presença e ausência do zumbido nos sujeitos avaliados ( $\mathrm{N}=6)$ 


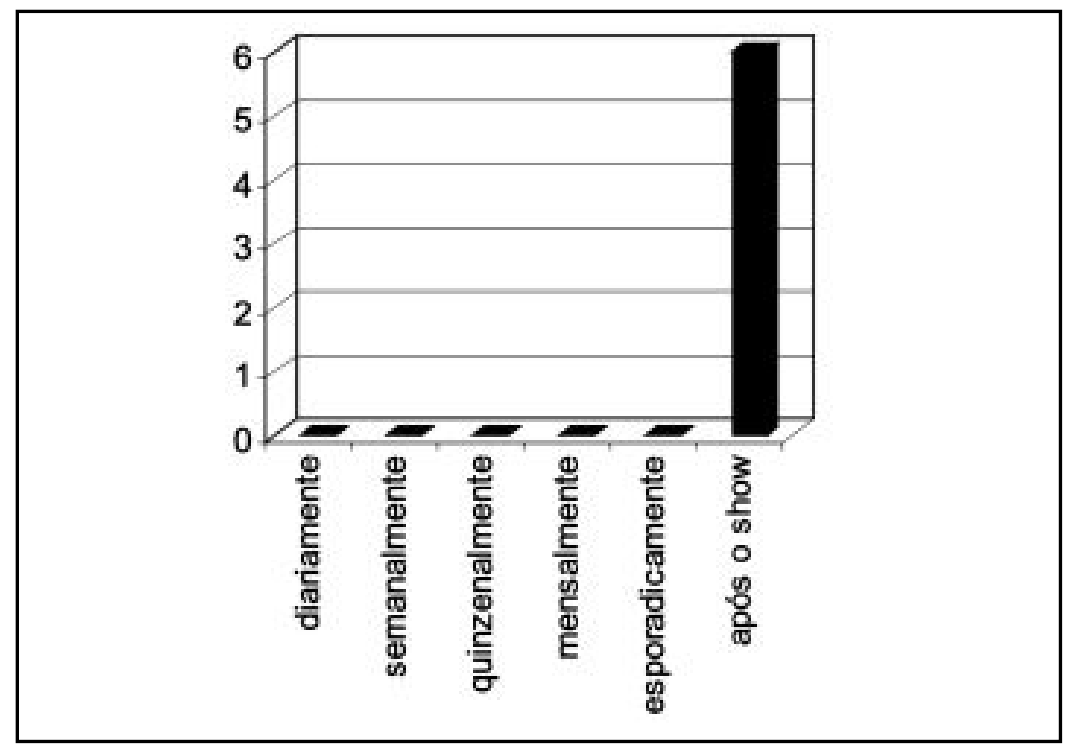

Figura 2 - Freqüência de aparecimento do zumbido nos sujeitos avaliados $(\mathrm{N}=6)$

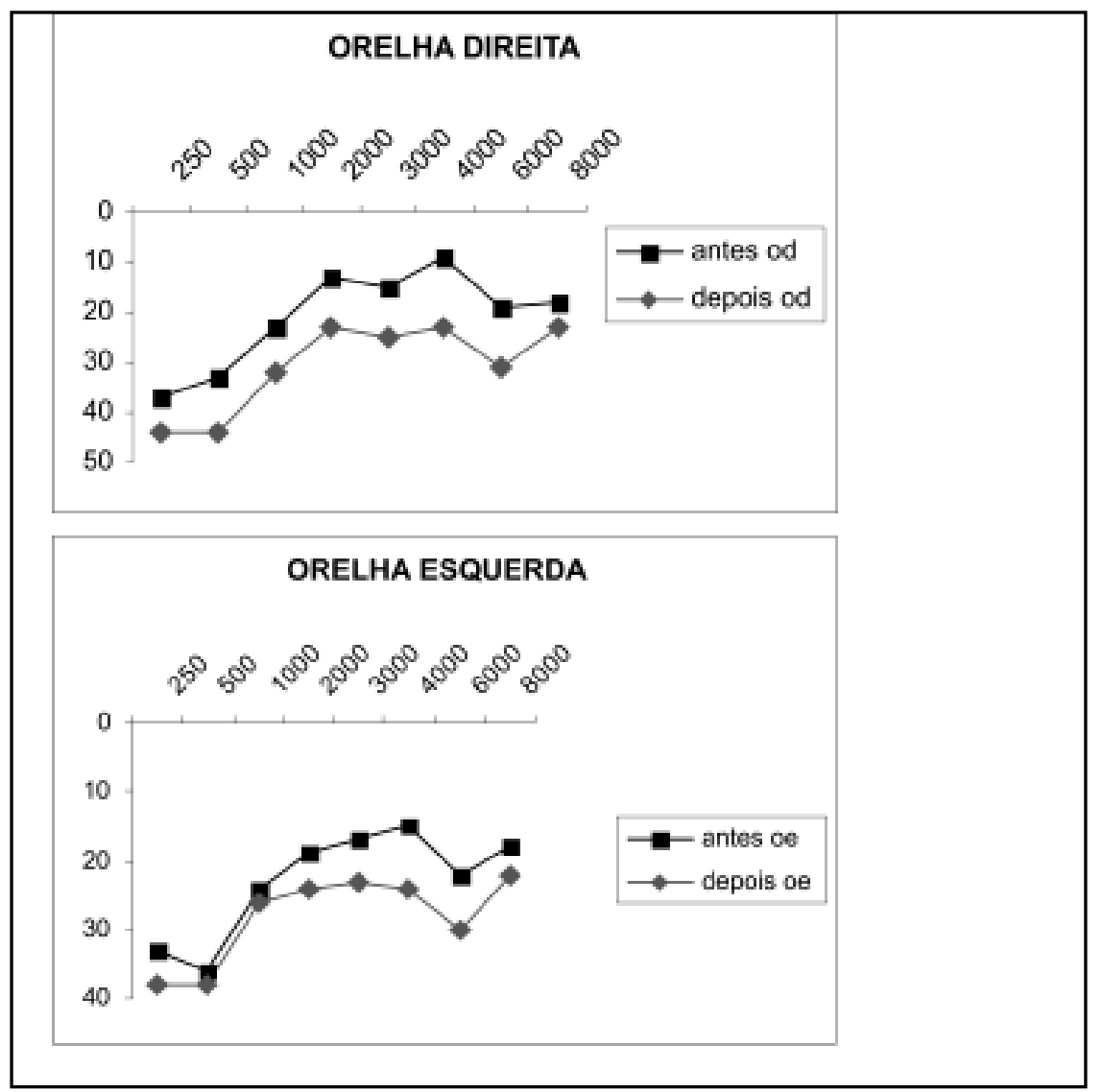

Figura 3 - Média audiométrica para as orelhas: direita e esquerda, antes e depois do show

\begin{tabular}{|l|c|c|c|c|c|c|c|c|}
\hline & $\mathbf{2 5 0}$ & $\mathbf{5 0 0}$ & $\mathbf{1 0 0 0}$ & $\mathbf{2 0 0 0}$ & $\mathbf{3 0 0 0}$ & $\mathbf{4 0 0 0}$ & $\mathbf{6 0 0 0}$ & $\mathbf{8 0 0 0 \mathrm { Hz }}$ \\
\hline $\mathrm{OD}$ & 1,63 & $2,28^{*}$ & 1,86 & $2,7^{*}$ & $2,59^{*}$ & $3,7^{*}$ & $2,86^{*}$ & 1,14 \\
\hline OE & 1,26 & 0,49 & 0,76 & 1,09 & 1,45 & $2,87^{*}$ & 1,46 & 0,93 \\
\hline
\end{tabular}

Valores significantes das freqüências por orelha na determinação dos níveis mínimos de audição, realizada antes e após o show de rock, de acordo com o teste $t$ Student 

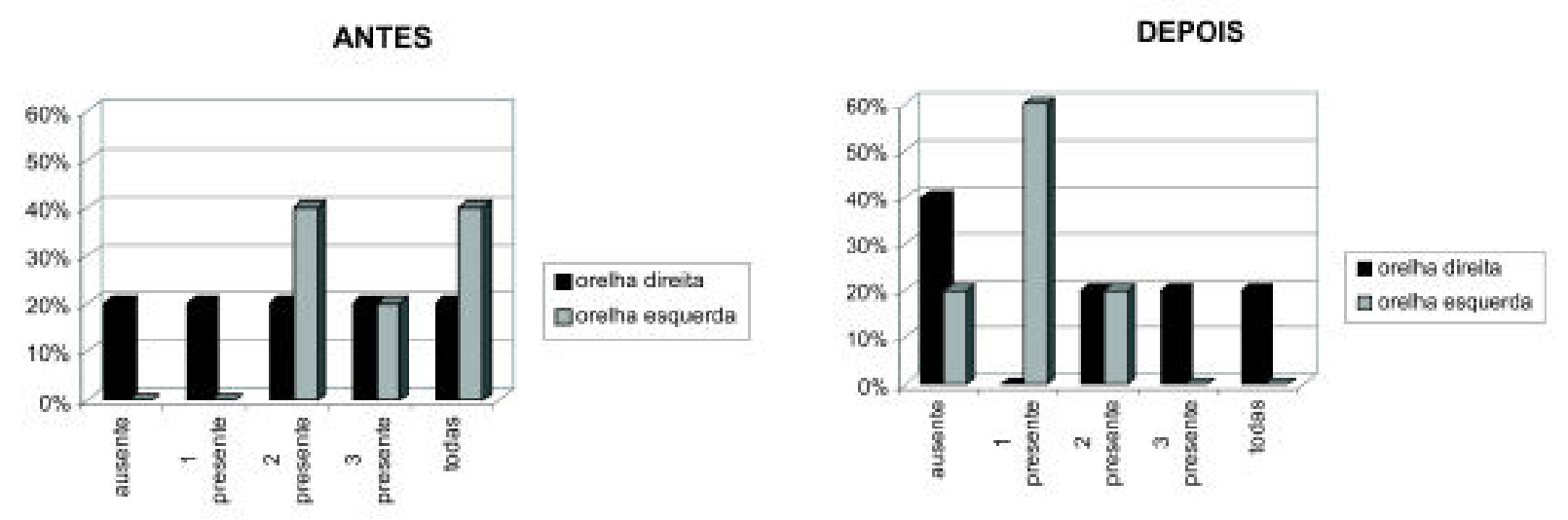

\begin{tabular}{|l|l|}
\hline OD & 0.19 \\
\hline $\mathrm{OE}$ & $3.64^{*}$ \\
\hline
\end{tabular}

Valores significantes por orelha no reflexo acústico, antes e após o show de rock, de acordo com o teste $t$ Student

\section{Figura 4 - Presença e ausência dos reflexos acústicos antes e após o show de rock}

\section{DISCUSSÃO}

Foi encontrado um valor de $98,5 \mathrm{~dB}$ de ruído durante o show, com o dosímetro posicionado no meio do palco, ou seja, um valor acima de $90 \mathrm{~dB}$, valor este lesivo e destrutivo ao sistema auditivo, concordando assim com o que foi relatado em outro estudo ${ }^{6}$.

Como o dosímetro foi posicionado no meio do palco, distante das caixas de som, não foi possível averiguar qual seria a intensidade da exposição sonora ao lado destas, não podendo assim comparar estes achados com os valores encontrados anteriormente em relato ${ }^{7}$. Acredita-se que os valores estariam acima dos encontrados, com o dosímetro localizado no meio do palco, sendo, portanto, mais prejudiciais aos músicos.

Com o objetivo de verificar a máxima mudança de limiar, como foi relatado anteriormente em outro estudo ${ }^{2}$, os exames foram realizados em até 30 minutos, após o término do show.

A música é um som prazeroso ao ouvido humano, porém este som possui potencial de ser descrito como ruído. Com os resultados desta pesquisa, é possível afirmar que a música pode transformar-se em um transtorno à saúde das pessoas, concordando, assim, com o que os autores afirmaram em outro artigo ${ }^{4}$.

Por esta razão, foi possível constatar que a música não é um som menos perigoso, por ser prazeroso, apesar de não ser o objeto de estudo, concordando, assim, com o que outro autor afirmou ${ }^{5}$, ou seja, sons desejados (prazerosos) como a música, apesar de serem menos perigosos do que os sons não desejados (como o ruído industrial), não deixam de ser um fator de risco para perdas auditivas.

Além disso, constatou-se que o aumento da exposição pode vir a trazer prejuízos à integridade física do músico, pois durante a anamnese, alguns músicos reclamaram de outros efeitos físicos, como a insônia após o trabalho, além da perda auditiva e do zumbido, concordando com o que foi afirmado em um artigo ${ }^{13}$.

Em 2004, foi estudada a diminuição da audição em DJ ${ }^{18}$, por meio da audiometria tonal e da aplicação de um questionário. A pesquisa foi realizada com o objetivo de constatar a mudança temporária de limiar desses profissionais, após uma noite de trabalho. Esta pesquisa comprovou que estes profissionais podem ser inseridos no grupo de trabalhadores com risco de perda auditiva induzida por níveis de pressão sonora elevados. Outros estudos ${ }^{18,19}$ mostraram, também, que o ambiente de discotecas é nocivo ao aparelho auditivo, verificando a mudança temporária do limiar auditivo nos freqüentadores, na saída do local.

Estudo semelhante ${ }^{20}$ foi realizado com músicos de rock de diversas modalidades musicais, em 1992. A pesquisa constatou que esses profissionais apresentavam uma mudança temporária de limiares auditivos após a exposição.

Nesta pesquisa, com o objetivo similar aos dois artigos relatados anteriormente, foi possível encontrar uma diferença estatisticamente significante dos níveis mínimos de audição, na freqüência de $500 \mathrm{~Hz}$ e nas freqüências compreendidas entre $3000 \mathrm{~Hz}$ e $6000 \mathrm{~Hz}$ na orelha direita, e na freqüência de $4000 \mathrm{~Hz}$, na orelha esquerda, antes 
e após o show de rock. Tais diferenças são semelhantes aos achados de outro autor ${ }^{11}$, revelando que a configuração das perdas auditivas induzidas por ruído mostra uma alteração primária nos limiares entre $3000 \mathrm{~Hz}$ e $6000 \mathrm{~Hz}$.

Com isso, é possível dizer que os músicos apresentaram, neste show analisado, uma mudança temporária de limiar, mudança esta descrita ${ }^{9}$ por uma redução na capacidade das células em perceberem a energia sonora que as atingem.

Esses achados concordam com um estudo semelhante ${ }^{12}$, ao afirmar que estas lesões vão desde a destruição total do aparelho auditivo até lesões de algumas de suas estruturas. Sendo assim, a mudança temporária de limiar pode se tornar uma perda auditiva induzida por ruído (PAIR), descrita anteriormente em outro artigo ${ }^{10}$.

Foram realizadas as medidas do reflexo acústico, pois de acordo com que foi afirmado anteriormente em outro artigo ${ }^{15}$, sobre a importância deste exame na determinação da mudança temporária de limiar, avaliação já definida previamente, ${ }^{14}$ como sendo um exame que registra a contração do músculo estapédico, na presença de um som de alta intensidade.

Não foi encontrado na literatura algo que justificasse a significância maior na orelha direita, na pesquisa dos níveis mínimos de audição, comprovando a mudança temporária de limiar, tampouco foi encontrada uma justificativa para as diferenças estatisticamente significantes, apenas na orelha esquerda, na pesquisa do reflexo acústico.
Acredita-se que este achado foi devido ao número reduzido da amostra, destacando-se, assim, a importância de serem realizados mais estudos sobre este assunto, com grupos amostrais maiores.

Com os achados da determinação dos níveis mínimos de audição e do reflexo acústico, pode-se concordar com o que foi afirmado em outras pesquisas ${ }^{8,20}$, que os músicos de bandas de rock são profissionais com risco de perda auditiva.

Concorda-se com o que outro estudo ${ }^{16}$ afirmou sobre a perda auditiva ocupacional ser irreversível, mas possível de prevenção e, como é de conhe-cimento, a mudança temporária de limiar freqüente pode desencadear uma perda auditiva ocupacional. Por esta razão, verifica-se a necessidade de mais estudos com estes profissionais e, também, a implantação de um programa de conservação auditiva.

Este estudo foi feito com um número restrito de músicos, de um determinado tipo de música; por esta razão, é necessária a realização de mais pesquisas com uma amostra maior, com outras modalidades musicais, em outras condições de apresentação.

\section{CONCLUSÃO}

Pode-se concluir que músicos expostos a níveis de pressão sonora intensos apresentaram alteração temporária do limiar e alteração do reflexo acústico e, por esta razão, são profissionais de risco para perda auditiva ocupacional.

\begin{abstract}
Purpose: verifying the temporary threshold shift in musicians after a high sound pressure level music exposure in a rock concert. Methods: the sample has been made up of six band's musicians, who underwent an occupational anamnesis, pure tone audiometry and acoustic reflex measurements, before and after the rock concert. The noise levels of the room and the stage were determined by means of a dosimeter. The results were compared and statically analyzed using the $t$ Student test with significance criterion of $p>0.05$. the noise level found in the room and stage during the concert was $98.5 \mathrm{~dB}$. As for the behavioral aspects related to the noise, tinnitus was the most prevalent complaint among the subjects. In pure tone audiometry, statistically significant differences were found in the hearing thresholds, before and after exposure, mostly in the right ear for the high frequencies. The results were also statistically significant at the frequencies between $2000 \mathrm{~Hz}$ and $6000 \mathrm{~Hz}$, for the right ear $(p=2.7 ; p=2.59 ; p=3.7$ e $p=2.86)$, and at the frequency of $4000 \mathrm{~Hz}$, for the left ear $(p=2.87)$. The right ear's acoustic reflex has shown the highest index of absence $(40 \%)$; however, we found statistically significant differences in the presence of the acoustic reflex, before and after exposure, only for the left ear $(p=3.64)$. Conclusion: musicians exposed to high sound pressure levels in rock concerts have shown temporary threshold shift at the pure tone audiometry and acoustic reflex measurements.
\end{abstract}

KEYWORDS: Audiology; Noise, Occupational; Hearing Tests; Hearing Loss 


\section{AGRADECIMENTOS}

Agradecemos ao profissionalismo e respeito ao nosso estudo à Banda Detonautas e dedicamos este trabalho ao músico Rodrigo Netto (Nettinho), guitarrista da banda, assassinado durante um assalto, em Junho de 2006, na cidade do Rio de Janeiro.

\section{REFERÊNCIAS}

1.Sataloff RT, Sataloff J. Occupational hearing loss. New York: Dekker; 1987.

2.Silva LF. Estudo sobre a exposição combinada entre ruído e vibração de corpo inteiro e os efeitos na audição de trabalhadores. [doutorado]. São Paulo (SP): Faculdade de Saúde Pública; Universidade de São Paulo; 2002.

3. Oliveira JAA. Audiovestibular toxicity of drugs. v. 1,2. Boca Raton FL: CC Press; 1989. 551 p.

4.Andrade AIA, Russo ICP, Lima MLLT et al. Avaliação auditiva em músicos de frevo e maracatu. Rev Bras Otorrinolaringol. 2002; 68(5):714-20.

5.Samelli AG, Schochat E. Perda auditiva induzida por nível de pressão sonora elevado em um grupo de músicos profissionais de rock-and-roll. Acta AWHO. 2000; 19(3):136-43.

6.Muniz L, Caldas N, Caldas Neto S, Lewis DR, Lessa F. Estudo das amplitudes das emissões otoacústicas em indivíduos expostos ao ruído de trios elétricos. An Fac Med Univ Fed Pernambuco. 2001; 46(1):28-31. 7. Jorge Júnior JJ, Monte Alegre AC. A audição dos jovens e sua relação com hábitos de exposição à música eletronicamente amplificada: introdução ao tema e uma revisão bibliográfica. Rev Bras Otorrinolaringol. 1995; 6(1):7-13.

8. Yassi A, Pollock N, Tran N, Cheang M. Risks to hearing from a rock concert. Can Fam Physician. 1993; 39:1045-50.
9.Souza MT. A reabilitação auditiva em motoristas de ônibus urbano, portadores de perda auditiva induzida por ruído (PAIR): proposta metodológica. [doutorado]. São Paulo (SP): Faculdade de Saúde Pública; Universidade de São Paulo; 2002.

10. Hausler R. The effects of acoustic overstimulation. Umsch. 2004; 61(1):21-9.

11. Pizarro G, Pizarro GU. Diagnóstico clínico pela audiometria ocupacional. Acta AWHO. 2000; 19(1):32-8.

12. Ugalde ACL, Dolci GEF, Magaña RC, González AM, Robles MI. Hipoacusia por ruído: um problema de salud y de conciencia pública. Rev Fac Med. 2000; 43(2):41-2.

13. Ferraz NM. Ruído e saúde. Mundo Saúde. 1996; 20(3):103-5.

14. Wever EG, Lawrence M. Physiological acoustics. Princeton: Princeton University Press; 1954.

15. Gerhardt KJ, Hepler Junior EL. Acoustic-reflex activity and behavioral thresholds following exposure to noise. J Acoust Soc Am. 1983; 74(1):109-14.

16. Kwitko A, Ferreira PG, França MT, Zanzini C, Steggiorin S. Perdas auditivas ocupacionais: análise de variáveis e diagnóstico. Rev Bras Med Otorrinolaringol. 1996; 3(3):151-64.

17. Rossi AG. Imitânciometria. In: Frota S. Fundamentos em fonoaudiologia: audiologia. 2. ed. Guanabara-Koogan; 2003. p. 77-86.

18. Bray A, Szymanski M, Mills R. Noise induced hearing loss in dance music disc jockeys and an examination of sound levels in nightclubs. J Laryngol Otol. 2004; 118 (2):123.

19. Wazen SR, Russo ICP. A study of hearing and of the auditory habits of youngsters of the city of Sorocaba - São Paulo. Pró-fono. 2004; 16(1):83-94. 20. Drake-Lee AB. Beyond music: auditory temporary threshold shift in rock musicians after a heavy metal concert. J R Soc Med. 1992; 85(10):617-9.
RECEBIDO EM: 08/02/2007

ACEITO EM: 15/07/2007

Endereço para correspondência:

Rua do Imperador 319 ap. 401

Petrópolis - RJ

CEP: 25620-002

Tel: (24) 22428202 / (24) 92514809

E-mail: marcela.pfeiffer@gmail.com 